\title{
Protecting against researcher bias in secondary data analysis: challenges and potential solutions
}

\author{
Jessie R. Baldwin ${ }^{1,2}$ (D) Jean-Baptiste Pingault ${ }^{1,2} \cdot$ Tabea Schoeler $^{1} \cdot$ Hannah M. Sallis ${ }^{3,4,5} \cdot$ Marcus R. Munafò ${ }^{3,4,6}$
}

Received: 19 October 2021 / Accepted: 28 December 2021

(c) The Author(s) 2022

\begin{abstract}
Analysis of secondary data sources (such as cohort studies, survey data, and administrative records) has the potential to provide answers to science and society's most pressing questions. However, researcher biases can lead to questionable research practices in secondary data analysis, which can distort the evidence base. While pre-registration can help to protect against researcher biases, it presents challenges for secondary data analysis. In this article, we describe these challenges and propose novel solutions and alternative approaches. Proposed solutions include approaches to (1) address bias linked to prior knowledge of the data, (2) enable pre-registration of non-hypothesis-driven research, (3) help ensure that pre-registered analyses will be appropriate for the data, and (4) address difficulties arising from reduced analytic flexibility in pre-registration. For each solution, we provide guidance on implementation for researchers and data guardians. The adoption of these practices can help to protect against researcher bias in secondary data analysis, to improve the robustness of research based on existing data.
\end{abstract}

Keywords Secondary data analysis $\cdot$ Pre-registration $\cdot$ Open science $\cdot$ Researcher bias

\section{Introduction}

Secondary data analysis has the potential to provide answers to science and society's most pressing questions. An abundance of secondary data exists-cohort studies, surveys, administrative data (e.g., health records, crime records,

Jessie R. Baldwin

j.baldwin@ucl.ac.uk

1 Department of Clinical, Educational and Health Psychology, Division of Psychology and Language Sciences, University College London, London WC1H 0AP, UK

2 Social, Genetic and Developmental Psychiatry Centre, Institute of Psychiatry, Psychology and Neuroscience, King's College London, London, UK

3 MRC Integrative Epidemiology Unit at the University of Bristol, Bristol Medical School, University of Bristol, Bristol, UK

4 School of Psychological Science, University of Bristol, Bristol, UK

5 Centre for Academic Mental Health, Population Health Sciences, University of Bristol, Bristol, UK

6 NIHR Biomedical Research Centre, University Hospitals Bristol NHS Foundation Trust and University of Bristol, Bristol, UK census data), financial data, and environmental data-that can be analysed by researchers in academia, industry, thirdsector organisations, and the government. However, secondary data analysis is vulnerable to questionable research practices (QRPs) which can distort the evidence base. These QRPs include p-hacking (i.e., exploiting analytic flexibility to obtain statistically significant results), selective reporting of statistically significant, novel, or "clean" results, and hypothesising after the results are known (HARK-ing [i.e., presenting unexpected results as if they were predicted]; [1]. Indeed, findings obtained from secondary data analysis are not always replicable [2, 3], reproducible [4], or robust to analytic choices [5, 6]. Preventing QRPs in research based on secondary data is therefore critical for scientific and societal progress.

A primary cause of QRPs is common cognitive biases that affect the analysis, reporting, and interpretation of data [7-10]. For example, apophenia (the tendency to see patterns in random data) and confirmation bias (the tendency to focus on evidence that is consistent with one's beliefs) can lead to particular analytical choices and selective reporting of "publishable" results [11-13]. In addition, hindsight bias (the tendency to view past events as predictable) can lead to HARK-ing, so that observed results appear more compelling. 
The scope for these biases to distort research outputs from secondary data analysis is perhaps particularly acute, for two reasons. First, researchers now have increasing access to high-dimensional datasets that offer a multitude of ways to analyse the same data [6]. Such analytic flexibility can lead to different conclusions depending on the analytical choices made $[5,14,15]$. Second, current incentive structures in science reward researchers for publishing statistically significant, novel, and/or surprising findings [16]. This combination of opportunity and incentive may lead researchersconsciously or unconsciously - to run multiple analyses and only report the most "publishable" findings.

One way to help protect against the effects of researcher bias is to pre-register research plans $[17,18]$. This can be achieved by pre-specifying the rationale, hypotheses, methods, and analysis plans, and submitting these to either a third-party registry (e.g., the Open Science Framework [OSF]; https://osf.io/), or a journal in the form of a Registered Report [19]. Because research plans and hypotheses are specified before the results are known, pre-registration reduces the potential for cognitive biases to lead to p-hacking, selective reporting, and HARK-ing [20]. While pre-registration is not necessarily a panacea for preventing QRPs (Table 1), meta-scientific evidence has found that pre-registered studies and Registered Reports are more likely to report null results [21-23], smaller effect sizes [24], and be replicated [25]. Pre-registration is increasingly being adopted in epidemiological research [26, 27], and is even required for access to data from certain cohorts (e.g., the Twins Early Development Study [28]). However, pre-registration (and other open science practices; Table 2) can pose particular challenges to researchers conducting secondary data analysis [29], motivating the need for alternative approaches and solutions. Here we describe such challenges, before proposing potential solutions to protect against researcher bias in secondary data analysis (summarised in Fig. 1).

Table 1 Limitations in the use of pre-registration to address QRPs

\begin{tabular}{lc}
\hline Limitation & Example \\
\hline Pre-registration may not prevent selective reporting/outcome switching & $\begin{array}{c}\text { The COMPare Trials Project [62] assessed outcome switching in } \\
\text { clinical trials published in the top 5 medical journals between } \\
\text { October 2015 and January 2016. Among 67 clinical trials, on aver- } \\
\text { age, each trial reported 58.2\% of its specified outcomes, and silently } \\
\text { added 5.3 new outcomes } \\
\text { Mathieu et al. [63] assessed 323 clinical trials published in 2008 in the } \\
\text { top 10 medical journals. } 45 \text { trials (13.9\%) were registered after the } \\
\text { completion of the study }\end{array}$ \\
$\begin{array}{l}\text { Pre-registration may be performed retrospectively after the results are } \\
\text { known }\end{array}$ & $\begin{array}{l}\text { Claesen et al. [57] assessed all pre-registered articles published in Psy- } \\
\text { chological Science and between February 2015 and November 2017. } \\
\text { Deviations from pre-registered protocols are common } \\
\text { disclosed the deviation }\end{array}$ \\
$\begin{array}{l}\text { Rubin [64] and Szollosi, Kellen [65] argue that formulating hypotheses } \\
\text { post-hoc (HARK-ing) is not problematic if they are deduced from } \\
\text { pre-existing theory or evidence, rather than induced from the current } \\
\text { results }\end{array}$ \\
\hline
\end{tabular}

Table 2 Challenges and potential solutions regarding sharing pre-existing data

\begin{tabular}{|c|c|}
\hline Challenge & Potential solutions \\
\hline $\begin{array}{l}\text { Data cannot be made open: } \\
\text { Many datasets cannot be publicly shared because of ethical and legal } \\
\text { requirements }\end{array}$ & $\begin{array}{l}\text { Share a synthetic dataset (a simulated dataset which mimics an origi- } \\
\text { nal dataset by preserving its statistical properties and associations } \\
\text { between variables). For a tutorial, see Quintana [66] } \\
\text { Provide specific instructions on how data can be accessed and links to } \\
\text { codebooks/data dictionaries with variable information [67] }\end{array}$ \\
\hline $\begin{array}{l}\text { Open data can lead to sequential testing problems: } \\
\text { If different researchers conduct similar statistical tests on a dataset } \\
\text { and do not correct for multiple testing, this increases the risk of false } \\
\text { positives [68] }\end{array}$ & $\begin{array}{l}\text { Test whether findings replicate in independent samples, as the chance of } \\
\text { two identical false positives occurring in independent samples is small } \\
\text { Ensure that the research question is distinct from prior studies on the } \\
\text { given dataset, to help ensure that proposed analyses are part of a dif- } \\
\text { ferent statistical family. Multiple analyses on a single dataset will not } \\
\text { lead to false positives if the analyses are part of different statistical } \\
\text { families }\end{array}$ \\
\hline
\end{tabular}


Fig. 1 Challenges in pre-registering secondary data analysis and potential solutions (according to researcher motivations). Note: In the "Potential solution" column, blue boxes indicate solutions that are researcher-led; green boxes indicate solutions that should be facilitated by data guardians

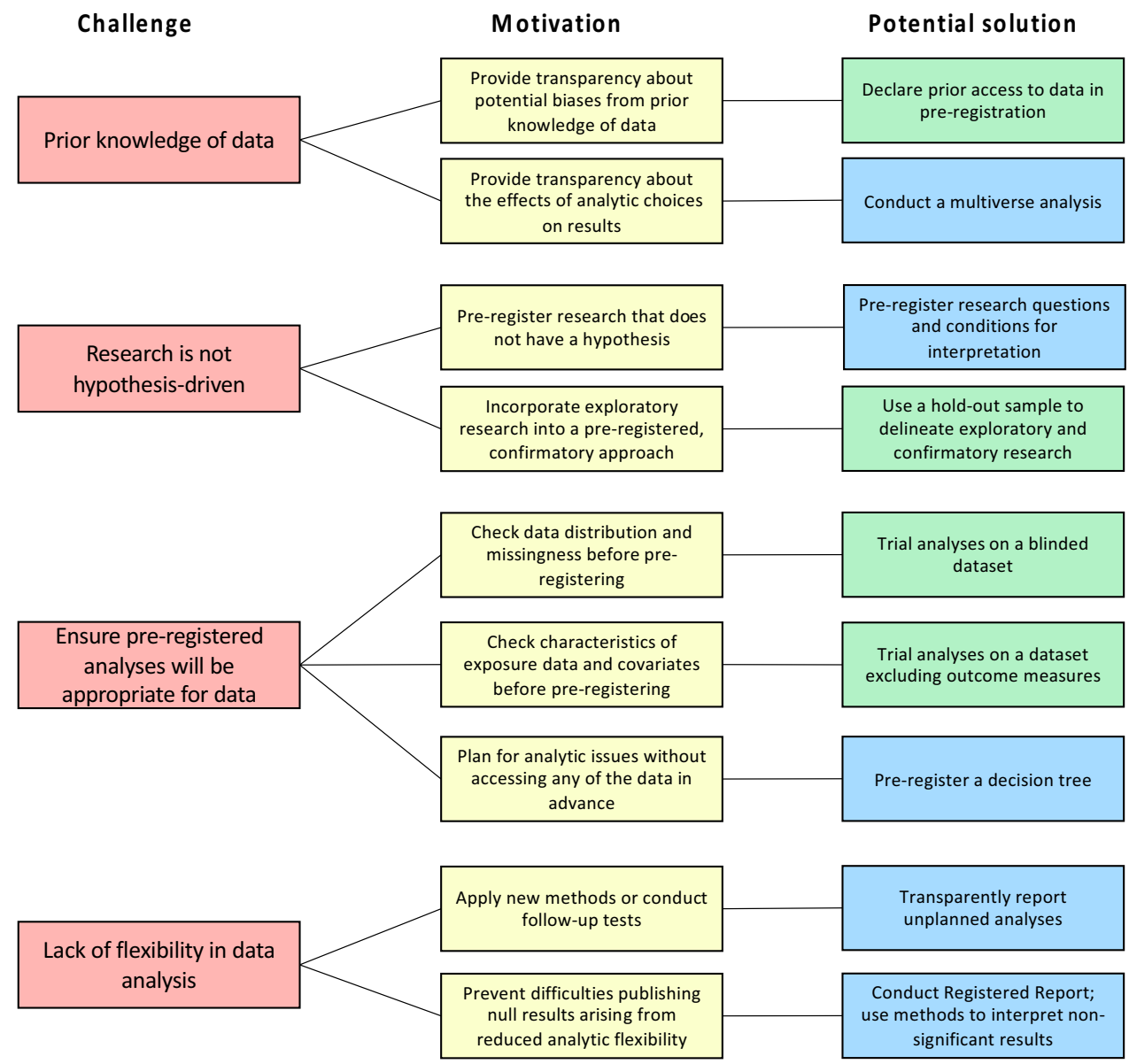

\section{Challenges of pre-registration for secondary data analysis}

\section{Prior knowledge of the data}

Researchers conducting secondary data analysis commonly analyse data from the same dataset multiple times throughout their careers. However, prior knowledge of the data increases risk of bias, as prior expectations about findings could motivate researchers to pursue certain analyses or questions. In the worst-case scenario, a researcher might perform multiple preliminary analyses, and only pursue those which lead to notable results (perhaps posting a pre-registration for these analyses, even though it is effectively post hoc). However, even if the researcher has not conducted specific analyses previously, they may be biased (either consciously or subconsciously) to pursue certain analyses after testing related questions with the same variables, or even by reading past studies on the dataset. As such, pre-registration cannot fully protect against researcher bias when researchers have previously accessed the data.

\section{Research may not be hypothesis-driven}

Pre-registration and Registered Reports are tailored towards hypothesis-driven, confirmatory research. For example, the OSF pre-registration template requires researchers to state "specific, concise, and testable hypotheses", while Registered Reports do not permit purely exploratory research [30], although a new Exploratory Reports format now exists [31]. However, much research involving secondary data is not focused on hypothesis testing, but is exploratory, descriptive, or focused on estimation-in other words, examining the magnitude and robustness of an association as precisely as possible, rather than simply testing a point null. Furthermore, without a strong theoretical background, hypotheses will be arbitrary and could lead to unhelpful inferences [32, 33], and so should be avoided in novel areas of research.

\section{Pre-registered analyses are not appropriate for the data}

With pre-registration, there is always a risk that the data will violate the assumptions of the pre-registered analyses 
[17]. For example, a researcher might pre-register a parametric test, only for the data to be non-normally distributed. However, in secondary data analysis, the extent to which the data shape the appropriate analysis can be considerable. First, longitudinal cohort studies are often subject to missing data and attrition. Approaches to deal with missing data (e.g., listwise deletion; multiple imputation) depend on the characteristics of missing data (e.g., the extent and patterns of missingness [34]), and so prespecifying approaches to dealing with missingness may be difficult, or extremely complex. Second, certain analytical decisions depend on the nature of the observed data (e.g., the choice of covariates to include in a multiple regression might depend on the collinearity between the measures, or the degree of missingness of different measures that capture the same construct). Third, much secondary data (e.g., electronic health records and other administrative data) were never collected for research purposes, so can present several challenges that are impossible to predict in advance [35]. These issues can limit a researcher's ability to pre-register a precise analytic plan prior to accessing secondary data.

\section{Lack of flexibility in data analysis}

Concerns have been raised that pre-registration limits flexibility in data analysis, including justifiable exploration [36-38]. For example, by requiring researchers to commit to a pre-registered analysis plan, pre-registration could prevent researchers from exploring novel questions (with a hypothesis-free approach), conducting follow-up analyses to investigate notable findings [39], or employing newly published methods with advantages over those pre-registered. While this concern is also likely to apply to primary data analysis, it is particularly relevant to certain fields involving secondary data analysis, such as genetic epidemiology, where new methods are rapidly being developed [40], and follow-up analyses are often required (e.g., in a genome-wide association study to further investigate the role of a genetic variant associated with a phenotype). However, this concern is perhaps over-stated - pre-registration does not preclude unplanned analyses; it simply makes it more transparent that these analyses are post hoc. Nevertheless, another understandable concern is that reduced analytic flexibility could lead to difficulties in publishing papers and accruing citations. For example, pre-registered studies are more likely to report null results [22, 23], likely due to reduced analytic flexibility and selective reporting. While this is a positive outcome for research integrity, null results are less likely to be published [13, 41, 42] and cited [11], which could disadvantage researchers' careers.

\section{Solutions}

In this section, we describe potential solutions to address the challenges involved in pre-registering secondary data analysis, including approaches to (1) address bias linked to prior knowledge of the data, (2) enable pre-registration of non-hypothesis-driven research, (3) ensure that preplanned analyses will be appropriate for the data, and (4) address potential difficulties arising from reduced analytic flexibility.

\section{Challenge: Prior knowledge of the data}

\section{Declare prior access to data}

To increase transparency about potential biases arising from knowledge of the data, researchers could routinely report all prior data access in a pre-registration [29]. This would ideally include evidence from an independent gatekeeper (e.g., a data guardian of the study) stating whether data and relevant variables were accessed by each co-author. To facilitate this process, data guardians could set up a central "electronic checkout" system that records which researchers have accessed data, what data were accessed, and when [43]. The researcher or data guardian could then provide links to the checkout histories for all co-authors in the pre-registration, to verify their prior data access. If it is not feasible to provide such objective evidence, authors could self-certify their prior access to the dataset and where possible, relevant variables-preferably listing any publications and in-preparation studies based on the dataset [29]. Of course, self-certification relies on trust that researchers will accurately report prior data access, which could be challenging if the study involves a large number of authors, or authors who have been involved on many studies on the dataset. However, it is likely to be the most feasible option at present as many datasets do not have available electronic records of data access. For further guidance on self-certifying prior data access when pre-registering secondary data analysis studies on a third-party registry (e.g., the OSF), we recommend referring to the template by Van den Akker, Weston [29].

The extent to which prior access to data renders preregistration invalid is debatable. On the one hand, even if data have been accessed previously, pre-registration is likely to reduce QRPs by encouraging researchers to commit to a pre-specified analytic strategy. On the other hand, pre-registration does not fully protect against researcher bias where data have already been accessed, and can lend added credibility to study claims, which 
may be unfounded. Reporting prior data access in a preregistration is therefore important to make these potential biases transparent, so that readers and reviewers can judge the credibility of the findings accordingly. However, for a more rigorous solution which protects against researcher bias in the context of prior data access, researchers should consider adopting a multiverse approach.

\section{Conduct a multiverse analysis}

A multiverse analysis involves identifying all potential analytic choices that could justifiably be made to address a given research question (e.g., different ways to code a variable, combinations of covariates, and types of analytic model), implementing them all, and reporting the results [44]. Notably, this method differs from the traditional approach in which findings from only one analytic method are reported. It is conceptually similar to a sensitivity analysis, but it is far more comprehensive, as often hundreds or thousands of analytic choices are reported, rather than a handful. By showing the results from all defensible analytic approaches, multiverse analysis reduces scope for selective reporting and provides insight into the robustness of findings against analytical choices (for example, if there is a clear convergence of estimates, irrespective of most analytical choices). For causal questions in observational research, Directed Acyclic Graphs (DAGs) could be used to inform selection of covariates in multiverse approaches [45] (i.e., to ensure that confounders, rather than mediators or colliders, are controlled for).

Specification curve analysis [46] is a form of multiverse analysis that has been applied to examine the robustness of epidemiological findings to analytic choices $[6,47]$. Specification curve analysis involves three steps: (1) identifying all analytic choices - termed "specifications", (2) displaying the results graphically with magnitude of effect size plotted against analytic choice, and (3) conducting joint inference across all results. When applied to the association between digital technology use and adolescent well-being [6], specification curve analysis showed that the (small, negative) association diminished after accounting for adequate control variables and recall bias - demonstrating the sensitivity of results to analytic choices.

Despite the benefits of the multiverse approach in addressing analytic flexibility, it is not without limitations. First, because each analytic choice is treated as equally valid, including less justifiable models could bias the results away from the truth. Second, the choice of specifications can be biased by prior knowledge (e.g., a researcher may choose to omit a covariate to obtain a particular result). Third, multiverse analysis may not entirely prevent selective reporting (e.g., if the full range of results are not reported), although pre-registering multiverse approaches (and specifying analytic choices) could mitigate this. Last, and perhaps most importantly, multiverse analysis is technically challenging (e.g., when there are hundreds or thousands of analytic choices) and can be impractical for complex analyses, very large datasets, or when computational resources are limited. However, this burden can be somewhat reduced by tutorials and packages which are being developed to standardise the procedure and reduce computational time [see 48, 49].

\section{Challenge: Research may not be hypothesis-driven}

\section{Pre-register research questions and conditions for interpreting findings}

Observational research arguably does not need to have a hypothesis to benefit from pre-registration. For studies that are descriptive or focused on estimation, we recommend preregistering research questions, analysis plans, and criteria for interpretation. Analytic flexibility will be limited by preregistering specific research questions and detailed analysis plans, while post hoc interpretation will be limited by pre-specifying criteria for interpretation [50]. The potential for HARK-ing will also be minimised because readers can compare the published study to the original pre-registration, where a-priori hypotheses were not specified.

Detailed guidance on how to pre-register research questions and analysis plans for secondary data is provided in Van den Akker's [29] tutorial. To pre-specify conditions for interpretation, it is important to anticipate - as much as possible - all potential findings, and state how each would be interpreted. For example, suppose that a researcher aims to test a causal relationship between $\mathrm{X}$ and $\mathrm{Y}$ using a multivariate regression model with longitudinal data. Assuming that all potential confounders have been fully measured and controlled for (albeit a strong assumption) and statistical power is high, three broad sets of results and interpretations could be pre-specified. First, an association between $\mathrm{X}$ and $\mathrm{Y}$ that is similar in magnitude to the unadjusted association would be consistent with a causal relationship. Second, an association between $\mathrm{X}$ and $\mathrm{Y}$ that is attenuated after controlling for confounders would suggest that the relationship is partly causal and partly confounded. Third, a minimal, nonstatistically significant adjusted association would suggest a lack of evidence for a causal effect of X on Y. Depending on the context of the study, criteria could also be provided on the threshold (or range of thresholds) at which the effect size would justify different interpretations [51], be considered practically meaningful, or the smallest effect size of interest for equivalence tests [52]. While researcher biases might still affect the pre-registered criteria for interpreting findings (e.g., toward over-interpreting a small effect size as meaningful), this bias will at least be transparent in the pre-registration. 


\section{Use a holdout sample to delineate exploratory and confirmatory research}

Where researchers wish to integrate exploratory research into a pre-registered, confirmatory study, a holdout sample approach can be used [18]. Creating a holdout sample refers to the process of randomly splitting the dataset into two parts, often referred to as 'training' and 'holdout' datasets. To delineate exploratory and confirmatory research, researchers can first conduct exploratory data analysis on the training dataset (which should comprise a moderate fraction of the data, e.g., 35\% [53]. Based on the results of the discovery process, researchers can pre-register hypotheses and analysis plans to formally test on the holdout dataset. This process has parallels with cross-validation in machine learning, in which the dataset is split and the model is developed on the training dataset, before being tested on the test dataset. The approach enables a flexible discovery process, before formally testing discoveries in a non-biased way.

When considering whether to use the holdout sample approach, three points should be noted. First, because the training dataset is not reusable, there will be a reduced sample size and loss of power relative to analysing the whole dataset. As such, the holdout sample approach will only be appropriate when the original dataset is large enough to provide sufficient power in the holdout dataset. Second, when the training dataset is used for exploration, subsequent confirmatory analyses on the holdout dataset may be overfitted (due to both datasets being drawn from the same sample), so replication in independent samples is recommended. Third, the holdout dataset should be created by an independent data manager or guardian, to ensure that the researcher does not have knowledge of the full dataset. However, it is straightforward to randomly split a dataset into a holdout and training sample and we provide example R code at: https://github. com/jr-baldwin/Researcher_Bias_Methods/blob/main/Holdo ut_script.md.

\section{Challenge: Pre-registered analyses are not appropriate for the data}

\section{Use blinding to test proposed analyses}

One method to help ensure that pre-registered analyses will be appropriate for the data is to trial the analyses on a blinded dataset [54], before pre-registering. Data blinding involves obscuring the data values or labels prior to data analysis, so that the proposed analyses can be trialled on the data without observing the actual findings. Various types of blinding strategies exist [54], but one method that is appropriate for epidemiological data is "data scrambling" [55]. This involves randomly shuffling the data points so that any associations between variables are obscured, whilst the variable distributions (and amounts of missing data) remain the same. We provide a tutorial for how to implement this in R (see https://github.com/jr-baldwin/Researcher_Bias_ Methods/blob/main/Data_scrambling_tutorial.md). Ideally the data scrambling would be done by a data guardian who is independent of the research, to ensure that the main researcher does not access the data prior to pre-registering the analyses. Once the researcher is confident with the analyses, the study can be pre-registered, and the analyses conducted on the unscrambled dataset.

Blinded analysis offers several advantages for ensuring that pre-registered analyses are appropriate, with some limitations. First, blinded analysis allows researchers to directly check the distribution of variables and amounts of missingness, without having to make assumptions about the data that may not be met, or spend time planning contingencies for every possible scenario. Second, blinded analysis prevents researchers from gaining insight into the potential findings prior to pre-registration, because associations between variables are masked. However, because of this, blinded analysis does not enable researchers to check for collinearity, predictors of missing data, or other covariances that may be necessary for model specification. As such, blinded analysis will be most appropriate for researchers who wish to check the data distribution and amounts of missingness before pre-registering.

\section{Trial analyses on a dataset excluding the outcome}

Another method to help ensure that pre-registered analyses will be appropriate for the data is to trial analyses on a dataset excluding outcome data. For example, data managers could provide researchers with part of the dataset containing the exposure variable(s) plus any covariates and/or auxiliary variables. The researcher can then trial and refine the analyses ahead of pre-registering, without gaining insight into the main findings (which require the outcome data). This approach is used to mitigate bias in propensity score matching studies [26, 56], as researchers use data on the exposure and covariates to create matched groups, prior to accessing any outcome data. Once the exposed and nonexposed groups have been matched effectively, researchers pre-register the protocol ahead of viewing the outcome data. Notably though, this approach could help researchers to identify and address other analytical challenges involving secondary data. For example, it could be used to check multivariable distributional characteristics, test for collinearity between multiple predictor variables, or identify predictors of missing data for multiple imputation.

This approach offers certain benefits for researchers keen to ensure that pre-registered analyses are appropriate for the observed data, with some limitations. Regarding benefits, researchers will be able to examine associations between 
variables (excluding the outcome), unlike the data scrambling approach described above. This would be helpful for checking certain assumptions (e.g., collinearity or characteristics of missing data such as whether it is missing at random). In addition, the approach is easy to implement, as the dataset can be initially created without the outcome variable, which can then be added after pre-registration, minimising burden on data guardians. Regarding limitations, it is possible that accessing variables in advance could provide some insight into the findings. For example, if a covariate is known to be highly correlated with the outcome, testing the association between the covariate and the exposure could give some indication of the relationship between the exposure and the outcome. To make this potential bias transparent, researchers should report the variables that they already accessed in the pre-registration. Another limitation is that researchers will not be able to identify analytical issues relating to the outcome data in advance of pre-registration. Therefore, this approach will be most appropriate where researchers wish to check various characteristics of the exposure variable(s) and covariates, rather than the outcome. However, a "mixed" approach could be applied in which outcome data is provided in scrambled format, to enable researchers to also assess distributional characteristics of the outcome. This would substantially reduce the number of potential challenges to be considered in pre-registered analytical pipelines.

\section{Pre-register a decision tree}

If it is not possible to access any of the data prior to preregistering (e.g., to enable analyses to be trialled on a dataset that is blinded or missing outcome data), researchers could pre-register a decision tree. This defines the sequence of analyses and rules based on characteristics of the observed data [17]. For example, the decision tree could specify testing a normality assumption, and based on the results, whether to use a parametric or non-parametric test. Ideally, the decision tree should provide a contingency plan for each of the planned analyses, if assumptions are not fulfilled. Of course, it can be challenging and time consuming to anticipate every potential issue with the data and plan contingencies. However, investing time into pre-specifying a decision tree (or a set of contingency plans) could save time should issues arise during data analysis, and can reduce the likelihood of deviating from the pre-registration.

\section{Challenge: Lack of flexibility in data analysis}

\section{Transparently report unplanned analyses}

Unplanned analyses (such as applying new methods or conducting follow-up tests to investigate an interesting or unexpected finding) are a natural and often important part of the scientific process. Despite common misconceptions, preregistration does not permit such unplanned analyses from being included, as long as they are transparently reported as post-hoc. If there are methodological deviations, we recommend that researchers should (1) clearly state the reasons for using the new method, and (2) if possible, report results from both methods, to ideally show that the change in methods was not due to the results [57]. This information can either be provided in the manuscript or in an update to the original pre-registration (e.g., on the third-party registry such as the OSF), which can be useful when journal word limits are tight. Similarly, if researchers wish to include additional follow-up analyses to investigate an interesting or unexpected finding, this should be reported but labelled as "exploratory" or "post-hoc" in the manuscript.

\section{Ensure a paper's value does not depend on statistically significant results}

Researchers may be concerned that reduced analytic flexibility from pre-registration could increase the likelihood of reporting null results [22, 23], which are harder to publish $[13,42]$. To address this, we recommend taking steps to ensure that the value and success of a study does not depend on a significant p-value. First, methodologically strong research (e.g., with high statistical power, valid and reliable measures, robustness checks, and replication samples) will advance the field, whatever the findings. Second, methods can be applied to allow for the interpretation of statistically non-significant findings (e.g., Bayesian methods [58] or equivalence tests, which determine whether an observed effect is surprisingly small $[52,59,60]$. This means that the results will be informative whatever they show, in contrast to approaches relying solely on null hypothesis significance testing, where statistically non-significant findings cannot be interpreted as meaningful. Third, researchers can submit the proposed study as a Registered Report, where it will be evaluated before the results are available. This is arguably the strongest way to protect against publication bias, as inprinciple study acceptance is granted without any knowledge of the results. In addition, Registered Reports can improve the methodology, as suggestions from expert reviewers can be incorporated into the pre-registered protocol.

\section{Conclusion}

Under a system that rewards novel and statistically significant findings, it is easy for subconscious human biases to lead to QRPs. However, researchers, along with data guardians, journals, funders, and institutions, have a responsibility to ensure that findings are reproducible and robust. While 
pre-registration can help to limit analytic flexibility and selective reporting, it involves several challenges for epidemiologists conducting secondary data analysis. The approaches described here aim to address these challenges (Fig. 1), to either improve the efficacy of pre-registration or provide an alternative approach to address analytic flexibility (e.g., a multiverse analysis). The responsibility in adopting these approaches should not only fall on researchers' shoulders; data guardians also have an important role to play in recording and reporting access to data, providing blinded datasets and holdout samples, and encouraging researchers to pre-register and adopt these solutions as part of their data request. Furthermore, wider stakeholders could incentivise these practices; for example, journals could provide a designated space for researchers to report deviations from the pre-registration, and funders could provide grants to establish best practice at the cohort level (e.g., data checkout systems, blinded datasets). Ease of adoption is key to ensure wide uptake, and we therefore encourage efforts to evaluate, simplify and improve these practices. Steps that could be taken to evaluate these practices are presented in Box 1.

More broadly, it is important to emphasise that researcher biases do not operate in isolation, but rather in the context of wider publication bias and a "publish or perish" culture. These incentive structures not only promote QRPs [61], but also discourage researchers from pre-registering and adopting other time-consuming reproducible methods. Therefore, in addition to targeting bias at the individual researcher level, wider initiatives from journals, funders, and institutions are required to address these institutional biases [7]. Systemic changes that reward rigorous and reproducible research will help researchers to provide unbiased answers to science and society's most important questions.

\section{Box 1. Evaluation of approaches}

To evaluate, simplify and improve approaches to protect against researcher bias in secondary data analysis, the following steps could be taken.

\section{Co-creation workshops to refine approaches}

To obtain feedback on the approaches (including on any practical concerns or feasibility issues) co-creation workshops could be held with researchers, data managers, and wider stakeholders (e.g., journals, funders, and institutions).

\section{Empirical research to evaluate efficacy of approaches}

To evaluate the effectiveness of the approaches in preventing researcher bias and/or improving pre-registration, empirical research is needed. For example, to test the extent to which the multiverse analysis can reduce selective reporting, comparisons could be made between effect sizes from multiverse analyses versus effect sizes from meta-analyses (of non-preregistered studies) addressing the same research question. If smaller effect sizes were found in multiverse analyses, it would suggest that the multiverse approach can reduce selective reporting. In addition, to test whether providing a blinded dataset or dataset missing outcome variables could help researchers develop an appropriate analytical protocol, researchers could be randomly assigned to receive such a dataset (or no dataset), prior to pre-registration. If researchers who received such a dataset had fewer eventual deviations from the pre-registered protocol (in the final study), it would suggest that this approach can help ensure that proposed analyses are appropriate for the data.

\section{Pilot implementation of the measures}

To assess the practical feasibility of the approaches, data managers could pilot measures for users of the dataset (e.g., required pre-registration for access to data, provision of datasets that are blinded or missing outcome variables). Feedback could then be collected from researchers and data managers via about the experience and ease of use.

Acknowledgements The authors are grateful to Professor George Davey for his helpful comments on this article.

Author contributions JRB and MRM developed the idea for the article. The first draft of the manuscript was written by JRB, with support from MRM and all authors commented on previous versions of the manuscript. All authors read and approved the final manuscript.

Funding J.R.B is funded by a Wellcome Trust Sir Henry Wellcome fellowship (grant $215917 / Z / 19 / Z$ ). J.B.P is a supported by the Medical Research Foundation 2018 Emerging Leaders $1^{\text {st }}$ Prize in Adolescent Mental Health (MRF-160-0002-ELP-PINGA). M.R.M and H.M.S work in a unit that receives funding from the University of Bristol and the UK Medical Research Council (MC_UU_00011/5, MC_UU_00011/7), and M.R.M is also supported by the National Institute for Health Research (NIHR) Biomedical Research Centre at the University Hospitals Bristol National Health Service Foundation Trust and the University of Bristol. 


\section{Declarations}

Conflict of interest Author declares that they have no conflict of interest.

Open Access This article is licensed under a Creative Commons Attribution 4.0 International License, which permits use, sharing, adaptation, distribution and reproduction in any medium or format, as long as you give appropriate credit to the original author(s) and the source, provide a link to the Creative Commons licence, and indicate if changes were made. The images or other third party material in this article are included in the article's Creative Commons licence, unless indicated otherwise in a credit line to the material. If material is not included in the article's Creative Commons licence and your intended use is not permitted by statutory regulation or exceeds the permitted use, you will need to obtain permission directly from the copyright holder. To view a copy of this licence, visit http://creativecommons.org/licenses/by/4.0/.

\section{References}

1. Kerr NL. HARKing: Hypothesizing after the results are known. Pers Soc Psychol Rev. 1998;2(3):196-217.

2. Border R, Johnson EC, Evans LM, et al. No support for historical candidate gene or candidate gene-by-interaction hypotheses for major depression across multiple large samples. Am J Psychiatry. 2019;176(5):376-87.

3. Duncan LE, Keller MC. A critical review of the first 10 years of candidate gene-by-environment interaction research in psychiatry. Am J Psychiatry. 2011;168(10):1041-9.

4. Seibold H, Czerny S, Decke S, et al. A computational reproducibility study of PLOS ONE articles featuring longitudinal data analyses. PLoS ONE. 2021;16(6):e0251194. https://doi.org/10. 1371/journal.pone.0251194.

5. Botvinik-Nezer R, Holzmeister F, Camerer CF, et al. Variability in the analysis of a single neuroimaging dataset by many teams. Nature. 2020;582:84-8.

6. Orben A, Przybylski AK. The association between adolescent well-being and digital technology use. Nat Hum Behav. 2019;3(2):173.

7. Munafò MR, Nosek BA, Bishop DV, et al. A manifesto for reproducible science. Nat Hum Behav. 2017;1(1):0021.

8. Nuzzo R. How scientists fool themselves-and how they can stop. Nature News. 2015;526(7572):182.

9. Bishop DV. The psychology of experimental psychologists: Overcoming cognitive constraints to improve research: The 47th Sir Frederic Bartlett lecture. Q J Exp Psychol. 2020;73(1):1-19.

10. Greenland S. Invited commentary: The need for cognitive science in methodology. Am J Epidemiol. 2017;186(6):639-45.

11. De Vries Y, Roest A, de Jonge P, Cuijpers P, Munafò M, Bastiaansen $\mathrm{J}$. The cumulative effect of reporting and citation biases on the apparent efficacy of treatments: The case of depression. Psychol Med. 2018;48(15):2453-5.

12. Nickerson RS. Confirmation bias: A ubiquitous phenomenon in many guises. Rev Gen Psychol. 1998;2(2):175-220.

13. Franco A, Malhotra N, Simonovits G. Publication bias in the social sciences: Unlocking the file drawer. Science. 2014;345(6203):1502-5.

14. Silberzahn R, Uhlmann EL, Martin DP, et al. Many analysts, one data set: Making transparent how variations in analytic choices affect results. Adv Methods Pract Psychol Sci. 2018;1(3):337-56.

15. Simmons JP, Nelson LD, Simonsohn U. False-positive psychology: Undisclosed flexibility in data collection and analysis allows presenting anything as significant. Psychol Sci. 2011;22(11):1359-66.

16. Metcalfe J, Wheat, K., Munafo, M., Parry, J. Research integrity: A landscape study: UK Research and innovation 2020.

17. Nosek BA, Ebersole CR, DeHaven AC, Mellor DT. The preregistration revolution. Proc Natl Acad Sci. 2018;115(11):2600-6.

18. Wagenmakers E-J, Wetzels R, Borsboom D, van der Maas HL, Kievit RA. An agenda for purely confirmatory research. Perspect Psychol Sci. 2012;7(6):632-8.

19. Chambers CD. Registered reports: A new publishing initiative at Cortex. Cortex. 2013;49(3):609-10.

20. Nosek BA, Beck ED, Campbell L, et al. Preregistration is hard, and worthwhile. Trends Cogn Sci. 2019;23(10):815-8.

21. Kaplan RM, Irvin VL. Likelihood of null effects of large NHLBI clinical trials has increased over time. PLoS One. 2015;10(8):e0132382.

22. Allen C, Mehler DM. Open science challenges, benefits and tips in early career and beyond. PLoS Biol. 2019;17(5):e3000246.

23. Scheel AM, Schijen MR, Lakens D. An excess of positive results: Comparing the standard psychology literature with registered reports. Adv Methods Pract Psychol Sci. 2021;4(2):25152459211007468.

24. Schäfer T, Schwarz MA. The meaningfulness of effect sizes in psychological research: differences between sub-disciplines and the impact of potential biases. Front Psychol. 2019;10:813.

25. Protzko J, Krosnick J, Nelson LD, et al. High replicability of newly-discovered social-behavioral findings is achievable. PsyArXiv. 2020. doi:https://doi.org/10.31234/osf.io/n2a9x

26. Small DS, Firth D, Keele L, et al. Protocol for a study of the effect of surface mining in central appalachia on adverse birth outcomes. arXiv.org. 2020

27. Deshpande SK, Hasegawa RB, Weiss J, Small DS. Protocol for an observational study on the effects of playing football in adolescence on mental health in early adulthood. arXiv preprint 2018

28. Twins Early Development Study. TEDS Data Access Policy: 6. Pre-registration of analysis. https://www.teds.ac.uk/researchers/ teds-data-access-policy\#preregistration. Accessed 18 March 2021

29. Van den Akker O, Weston SJ, Campbell L, et al. Preregistration of secondary data analysis: a template and tutorial. PsyArXiv. 2019. doi:https://doi.org/10.31234/osf.io/hvfmr

30. Chambers C, Tzavella L. Registered reports: past, present and future. MetaArXiv. 2020. doi:https://doi.org/10.31222/osf.io/ 43298

31. McIntosh RD. Exploratory reports: A new article type for cortex. Cortex. 2017;96:A1-4.

32. Scheel AM, Tiokhin L, Isager PM, Lakens D. Why hypothesis testers should spend less time testing hypotheses. Perspect Psychol Sci. 2020;16(4):744-55.

33. Colhoun HM, McKeigue PM, Smith GD. Problems of reporting genetic associations with complex outcomes. Lancet. 2003;361(9360):865-72.

34. Hughes RA, Heron J, Sterne JAC, Tilling K. Accounting for missing data in statistical analyses: Multiple imputation is not always the answer. Int J Epidemiol. 2019;48(4):1294-304. https://doi.org/ 10.1093/ije/dyz032.

35. Goldstein BA. Five analytic challenges in working with electronic health records data to support clinical trials with some solutions. Clin Trials. 2020;17(4):370-6.

36. Goldin-Meadow S. Why preregistration makes me nervous. APS Observer. 2016;29(7).

37. Lash TL. Preregistration of study protocols is unlikely to improve the yield from our science, but other strategies might. Epidemiology. 2010;21(5):612-3. https://doi.org/10.1097/EDE.0b013e3181 e9bba6. 
38. Lawlor DA. Quality in epidemiological research: should we be submitting papers before we have the results and submitting more hypothesis-generating research? Int J Epidemiol. 2007;36(5):940-3.

39. Vandenbroucke JP. Preregistration of epidemiologic studies: An ill-founded mix of ideas. Epidemiology. 2010;21(5):619-20.

40. Pingault J-B, O'reilly PF, Schoeler T, Ploubidis GB, Rijsdijk F, Dudbridge F. Using genetic data to strengthen causal inference in observational research. Nat Rev Genet. 2018;19(9):566.

41. Fanelli D. Negative results are disappearing from most disciplines and countries. Scientometrics. 2012;90(3):891-904.

42. Greenwald AG. Consequences of prejudice against the null hypothesis. Psychol Bull. 1975;82(1):1.

43. Scott KM, Kline M. Enabling confirmatory secondary data analysis by logging data checkout. Adv Methods Pract Psychol Sci. 2019;2(1):45-54. https://doi.org/10.1177/2515245918815849.

44. Steegen S, Tuerlinckx F, Gelman A, Vanpaemel W. Increasing transparency through a multiverse analysis. Perspect Psychol Sci. 2016;11(5):702-12.

45. Del Giudice M, Gangestad SW. A traveler's guide to the multiverse: Promises, pitfalls, and a framework for the evaluation of analytic decisions. Adv Methods Pract Psychol Sci. 2021;4(1):2515245920954925

46. Simonsohn U, Simmons JP, Nelson LD. Specification curve: descriptive and inferential statistics on all reasonable specifications. SSRN. 2015. https://doi.org/10.2139/ssrn.2694998.

47. Rohrer JM, Egloff B, Schmukle SC. Probing birth-order effects on narrow traits using specification-curve analysis. Psychol Sci. 2017;28(12):1821-32.

48. Masur P. How to do specification curve analyses in R: Introducing 'specr'. 2020. https://philippmasur.de/2020/01/02/how-to-do-speci fication-curve-analyses-in-r-introducing-specr/. Accessed 23rd July 2020.

49. Masur PK, Scharkow M. specr: Conducting and visualizing specification curve analyses: R package. (2020).

50. Kiyonaga A, Scimeca JM. Practical considerations for navigating registered reports. Trends Neurosci. 2019;42(9):568-72.

51. McPhetres J. What should a preregistration contain? PsyArXiv. (2020).

52. Lakens D. Equivalence tests: A practical primer for t tests, correlations, and meta-analyses. Soc Psychol Personal Sci. 2017;8(4):355-62.

53. Anderson ML, Magruder J. Split-sample strategies for avoiding false discoveries: National Bureau of Economic Research2017. Report No.: 0898-2937.

54. MacCoun R, Perlmutter S. Blind analysis: Hide results to seek the truth. Nature. 2015;526(7572):187-9.
55. MacCoun R, Perlmutter S. Blind analysis as a correction for confirmatory bias in physics and in psychology. Psychological science under scrutiny 2017. p. 295-322.

56. Rubin DB. The design versus the analysis of observational studies for causal effects: Parallels with the design of randomized trials. Stat Med. 2007;26(1):20-36.

57. Claesen A, Gomes SLBT, Tuerlinckx F, Vanpaemel W. Preregistration: Comparing dream to reality. 2019.

58. Schönbrodt FD, Wagenmakers E-J. Bayes factor design analysis: Planning for compelling evidence. Psychon Bull Rev. 2018;25(1):128-42.

59. Lakens D, Scheel AM, Isager PM. Equivalence testing for psychological research: A tutorial. Adv Methods Pract Psychol Sci. 2018;1(2):259-69.

60. Lakens D, McLatchie N, Isager PM, Scheel AM, Dienes Z. Improving inferences about null effects with Bayes factors and equivalence tests. J Gerontol Ser B. 2020;75(1):45-57.

61. Gopalakrishna G, ter Riet G, Vink G, Stoop I, Wicherts J, Bouter L. Prevalence of questionable research practices, research misconduct and their potential explanatory factors: a survey among academic researchers in The Netherlands. 2021.

62. Goldacre B, Drysdale, H., Powell-Smith, A., Dale, A., Milosevic, I., Slade, E., Hartley, H., Marston, C., Mahtani, K., Heneghan, C. The compare trials project. 2021. https://compare-trials.org. Accessed 23rd July 2020.

63. Mathieu S, Boutron I, Moher D, Altman DG, Ravaud P. Comparison of registered and published primary outcomes in randomized controlled trials. JAMA. 2009;302(9):977-84.

64. Rubin M. Does preregistration improve the credibility of research findings? arXiv preprint 2020.

65. Szollosi A, Kellen D, Navarro D, et al. Is preregistration worthwhile? Cell. 2019.

66. Quintana DS. A synthetic dataset primer for the biobehavioural sciences to promote reproducibility and hypothesis generation. Elife. 2020;9:e53275.

67. Weston SJ, Ritchie SJ, Rohrer JM, Przybylski AK. Recommendations for increasing the transparency of analysis of preexisting data sets. Adv Methods Pract Psychol Sci. 2019;2(3):214-27.

68. Thompson WH, Wright J, Bissett PG, Poldrack RA. Metaresearch: dataset decay and the problem of sequential analyses on open datasets. Elife. 2020;9:e53498.

Publisher's Note Springer Nature remains neutral with regard to jurisdictional claims in published maps and institutional affiliations. 